\title{
A microarray for microRNA profiling in mouse testis tissues
}

\author{
Naihong Yan ${ }^{1,2}$, Yilu Lu ${ }^{1}$, Huaqin Sun ${ }^{1}$, Dachang Tao ${ }^{1}$, Sizhong Zhang ${ }^{1}$, Wenying Liu ${ }^{1}$ \\ and Yongxin $\mathrm{Ma}^{1}$ \\ ${ }^{1}$ Division of Human Morbid Genomics, State Key Laboratory of Biotherapy, Department of Medical Genetics, \\ West China Hospital, Sichuan University, Renmin Nanlu, Section 3, No. 17, Chengdu 610041, People's Republic \\ of China and ${ }^{2}$ Laboratory of Ophthalmology, West China Hospital, Sichuan University, Chengdu 610041, People's \\ Republic of China
}

Correspondence should be addressed to Y Ma; Email: mayongxing@263.net

\begin{abstract}
MicroRNAs (miRNAs) are short non-coding RNA molecules playing regulatory roles by repressing translation or cleaving RNA transcripts. Recent studies indicate that miRNAs are mechanistically involved in the development of mammalian spermatogenesis. However, little work has been done to compare the miRNA expression patterns between immature and mature mouse testes. Here, we employed a miRNA microarray to detect 892 miRNAs in order to evaluate the expression patterns of miRNA. The expression of 19 miRNAs was significantly different between immature and mature individuals. Fourteen miRNAs were significantly upregulated and five miRNAs were downregulated in immature mice and this result was further confirmed by a quantitative real-time RT-PCR assay. Many target genes involved in spermatogenesis are predicted by MiRscan performing miRNA target scanning. Our data indicated specific miRNAs expression in immature mouse testis and suggested that miRNAs have a role in regulating spermatogenesis.
\end{abstract}

Reproduction (2007) 134 73-79

\section{Introduction}

Spermatogenesis is the cellular process that produces mature male germ cells, which takes place within the seminiferous tubules of the testis of the sexually mature male. During spermatogenesis, post-transcriptional control plays an essential role for spermatid differentiation (Braun 1998) and can be mediated by sequences, such as microRNAs (miRNA)-targeting sequences, in the $5^{\prime}$ - and $3^{\prime}$-UTRs of miRNA.

miRNAs are small non-coding RNAs (typically 19-23 nucleotides) that play important roles in regulating posttranscriptional translation. The first discovered miRNA, lin-4, is involved in developmental timing in the nematode Caenorhabditis elegans (Lee et al. 1993). After that, hundreds of miRNAs have been discovered and identified in plants and animals. Many miRNAs are conserved and it may regulate up to $30 \%$ of genes (Lewis et al. 2003). About $68 \%$ of miRNAs are expressed in a highly tissue-specific manner (Wienholds et al. 2005), for example, miR-1 is a muscle- and heart-specific miRNA (Zhao et al. 2005). It is indicated that miRNAs play essential roles in the regulation of gene expression during development. During mouse spermatogenesis, evidence is emerging that miRNAs have a major function in translational regulation (Kuramochi-Miyagawa et al. 2004). One such miRNA, miR-122a, was suggested targeting a reporter mRNA containing sequences from the $3^{\prime}$-UTR of the transition protein 2 (Tnp2), Tnp2 is a post-transcriptionally regulated testis-specific gene involved in chromatin remodeling during mouse spermatogenesis (Yu et al. 2005). However, although thousands of miRNAs have been identified from mammalian somatic tissues, little is known regarding their expression in germ cells.

To explore the expression pattern of miRNA in spermatogenesis, we employed microarray technique on sexually immature and mature mouse testes and examined high-throughput miRNA expression; and the differences in expression levels were confirmed by quantitative RT-PCR. In this study, we analyzed 892 miRNAs and found that the levels of 19 miRNAs changed significantly between immature and mature mouse testes. The expression of 14 miRNAs was significantly enhanced, while the expression of 5 miRNAs greatly decreased in immature mouse testis. In this study, we use computational methods to identify common targets of miRNA and analyze the relationship between those putative genes and spermatogenesis. Our data indicate a pattern of miRNAs expression specific to 
immature mouse testis and suggest that miRNAs are able to regulate spermatogenesis.

\section{Materials and Methods}

\section{Animals}

Male Balb/c mice used in this study were obtained from the Experimental Animal Center of Sichuan University (Chengdu, China). All the animals were kept and bred in facilities of a standard conforming to UK Home Office requirements. Testes were obtained from 1-week-old immature Balb/c mice $(10.0 \pm 0.8 \mathrm{~g}$; marked $I)$ and 7 -week-old mature mice $(35.0 \pm 1.4 \mathrm{~g}$; marked $M$ ). Each group has eight individual samples. All the measures taken for the mice were in accordance with approved guidelines (Guidelines for the Care and Use of Laboratory Animals) established by the Chinese Council on Animal Care and in accordance with UK legal requirements.

\section{RNA extraction}

Animals were killed by cervical dislocation after $\mathrm{CO}_{2}$ asphyxiation. Whole testes were removed from animals and were immediately snap-frozen in liquid nitrogen, ground to a fine powder in a mortar that was pre-cooled with liquid nitrogen, and then Trizol reagent (Invitrogen) was added to continue grinding. Total RNA was extracted by Trizol reagent. All the procedures were carried out according to manufacturer's protocol.

\section{miRNA microarray analysis}

miRNA microarrays were obtained from CapitalBio Corporation (Beijing, China), corresponding to current release of Sanger miRNAs database (http://microrna. sanger.ac.uk, October 2005). The individual oligonucleotide probe was printed in triplicate on chemical modification glass slides and $21 \times 21$ spot configuration of each subarray. The spot diameter was $130 \mu \mathrm{m}$, and distance from center to center was $185 \mu \mathrm{m}$. miRNAs were enriched from total RNA extracted from samples I and $M$ with mirVana miRNA Isolation Kit (Ambion, Foster City, CA, USA) and labeled with mirVana Array Labeling Kit (Ambion). Labeled miRNAs were used for hybridization on each miRNA microarray containing 509 probes in triplicate, corresponding to 892 human, rat, and mouse miRNAs, to determine differential expression between $I$ and $M$ samples (Thomson et al. 2004). This procedure was repeated twice.

The miRNA microarray from CapitalBio Corporation was single-channel fluorescence chip; all oligonucleotide probes were labeled with Cy3 fluorescent dyes (green color). Fluorescence scanning used a double-channel laser scanner (LuxScan 10K/A, CapitalBio). Then, the figure signal was transformed to digital signal using image analysis software (LuxScan3.0, CapitalBio). Signal intensities for each spot were calculated by subtracting local background from total intensities. Raw data were normalized and analyzed using the Significance Analysis of Microarrays (SAM, version 2.1, Stanford University, CA, USA) software.

\section{Gene-specific RT}

DNase-treated total RNA ( $2 \mu \mathrm{g})$ was reverse transcribed to cDNA with gene-specific RT primers, using the RevertAid First Strand cDNA Synthesis Kit (MBI Fermentas, Vilnius, Lithuania). A total of 19 genespecific stem-loop RT primers were designed according to miRNAs sequences listed in the Sanger miRBase (microRNA sequences, targets, and gene nomenclature; Supplementary Table 1 which can be viewed online at www.reproduction-online.org/supplemental/).

Each reaction mixture of RT contained $2 \mu \mathrm{g}$ RNA sample, $50 \mathrm{nM}$ gene-specific stem-loop RT primer (U6 use random primers), $1 \times \mathrm{RT}$ buffer, $0.25 \mathrm{mM}$ of each of dNTPs, $200 \cup$ RevertAid M-MuLV Reverse Transcriptase, $20 \cup$ RNase inhibitor, and deionized water to a total volume of $20 \mu \mathrm{l}$. The reactions were incubated in an Applied Biosystems 9700 Thermocycler (Applied Biosystems, Foster City, CA, USA) in a 96-well plate for $30 \mathrm{~min}$ at $16^{\circ} \mathrm{C}, 30 \mathrm{~min}$ at $42^{\circ} \mathrm{C}, 5 \mathrm{~min}$ at $85^{\circ} \mathrm{C}$, and then held at $4{ }^{\circ} \mathrm{C}$. All RT reactions, including negative controls, were repeated thrice.

\section{Real-time PCR for miRNA precursors}

Gene-specific PCR forward primers were designed according to miRNA sequences, and a universal PCR reverse primer (Chen et al. 2005). The expression of the U6 small nuclear RNA (NR_003027) gene was used as an internal control (Schmittgen et al. 2004). All the miRNA-specific primers and U6 primer are listed in the Supplementary Table 2 which can be viewed online at www.reproduction-online.org/supplemental/.

The expression of the miRNA precursors was determined using quantitative real-time PCR as described earlier (Schmittgen et al. 2004) with several modifications. Standard curves of cDNA were composed of four tenfold dilutions of sample I cDNA. Quantitative real-time PCR was conducted using an FTC 2000 realtime PCR instrument (Funglyn, Toronto, Canada). Each reaction mixture contained $1 \times \mathrm{PCR}$ buffer, $2.5 \mathrm{mM}$ $\mathrm{MgCl}_{2}, 0.25 \mathrm{mM}$ of each of dNTPs, $1.5 \cup$ DNA polymerase, $1.5 \mu \mathrm{M}$ forward primer, $0.7 \mu \mathrm{M}$ reverse primer, $1 \times$ SYBR Green I, $2 \mu \mathrm{l}$ cDNA, and deionized water was added to a total volume of $30 \mu \mathrm{l}$. Unless indicated, all reagents used in this procedure were purchased from MBI Company (MBI Fermentas).

Cycling parameters were $95^{\circ} \mathrm{C}$ for $5 \mathrm{~min}$ to denature DNA templates, then 30 cycles of $95^{\circ} \mathrm{C}$ for $10 \mathrm{~s}$, and 
$60{ }^{\circ} \mathrm{C}$ for $1 \mathrm{~min}$, with a final recording step of $78{ }^{\circ} \mathrm{C}$ for $20 \mathrm{~s}$ to prevent any primer-dimer formation (Peirson et al. 2003). Melting curves were performed using Dissociation Curves software (Funglyn) to ensure only a single product was amplified, and samples were also run on a $3 \%$ agarose gel to confirm specificity. All reactions including negative controls were repeated thrice.

\section{Data analysis}

Relative quantification was conduced using amplification efficiencies derived from cDNA standard curves and obtained relative gene expression (Peirson et al. 2003). Data were analyzed initially using FTC2000 4.0 software (Funglyn). The relative expression of each miRNA to U6 RNA was described using the equation

$R=\frac{\left(E_{\mathrm{miRNA}}\right)^{\Delta \mathrm{CT}_{\mathrm{miRNA}}}}{\left(E_{\mathrm{U} 6}\right)^{\Delta \mathrm{CT}_{\mathrm{U} 6}}}$

where $R$ is the relative expression, $E$ is the amplification efficiency, CT is the threshold cycle, and $\Delta C T=(C T S a m p l e$ I-CTSample M). Amplification efficiency can then be calculated from the slope: Efficiency $=10(1 /$ slope $)-1$.

\section{Results}

\section{miRNA microarray analysis}

Having resulted in profiling expression of thousands of genes simultaneously, microarray assay provides a powerful tool for analyzing both miRNA expression patterns and quantitative expression levels. This technology is much more efficient than those outmoded time-consuming methods, and is becoming the broadest miRNA research tool available (Calin et al. 2004).

The mature/immature status of the samples was confirmed by examination of sperm smear for mature sperm. RNA gel electrophoresis presented that the quality of the RNA was good. The microarray contained
509 degenerated oligonucleotide probes generated from 892 miRNAs (435 human, include nature predicted 122 miRNAs, 261 mouse, and 196 rat; Xie et al. 2005). All of the oligonucleotide probes were repeated thrice in one microarray, and each of the four subarrays contained 16 controls (Zip5, Zip13, Zip15, Zip21, Zip23, Zip25, Y2, Y3, U6, New-U2-R, tRNA-R, has-let-7a, has-let-7b, has-let-7c, 50\% DMSO (dimethyl Sulphoxide), and Hex). For increased confidence, we repeated each microarray assay twice. A t-test statistical analysis and scatter diagrams of all spots showed that the reproducibility and reliability were good (Fig. $1 ; P>0.05$ ).

Class comparison and SAM were performed to identify differences in miRNA expression between $I$ and $M$ samples. SAM calculated out a score for each gene on the basis of the change in expression relative to the S.D. of all measurements (Tusher et al. 2001, He et al. 2005); the results are shown in Table 1.

Pairwise significance analysis of the microarray data indicated that 14 miRNA genes were significantly overexpressed in sexually immature mouse testis (sample I) with fold changes $>2$. Underexpression appeared only in five miRNAs (mmu-miR-34a, mmu-miR-29b, hsa-miR449, rno-miR-34b, and mmu-miR-34c). Three miRNAs (hsa-miR-495, hsa-miR-181d, and rno-miR-34b) did not have any corresponding mouse miRNAs.

\section{Real-time PCR for miRNA precursors}

To verify the accuracy of the microarray results above, we used quantitative real-time RT-PCR to measure the expression levels of individual miRNAs. Since miRNA precursors are short, it is difficult to amplify and quantify these short RNA targets by PCR method. A novel scheme for PCR assays was applied for specificity and sensitivity; this used stem-loop RT primers, which can specifically quantify miRNA expression levels over existing conventional detection methods (Chen et al. 2005).
A
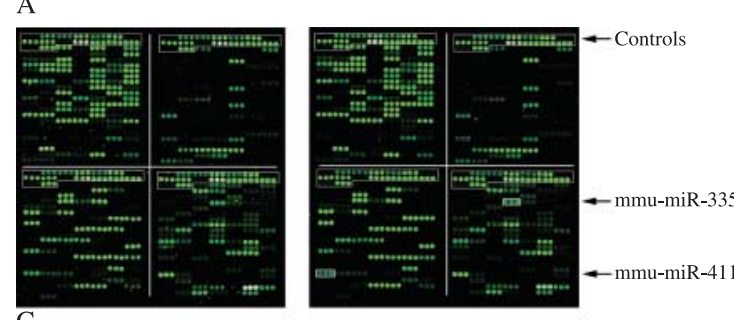

B

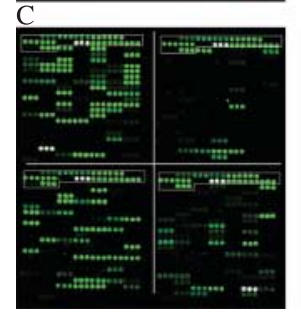

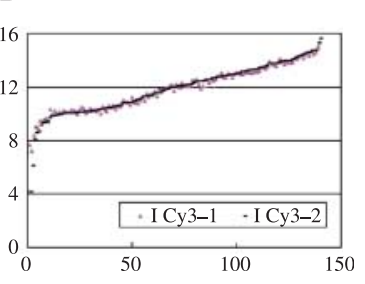

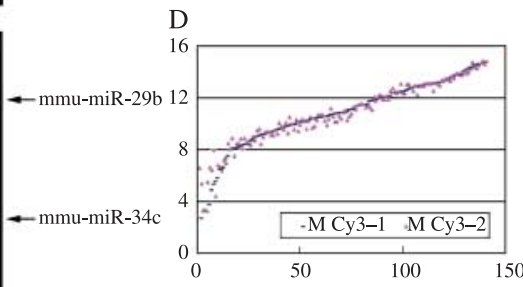

Figure 1 Expression pattern of miRNA in immature and mature mouse testis. A: hybridization signal of sample I Cy3; B: scatter diagram showing high reproducibility between the replicate experiments of sample l; C: hybridization signal of sample $M$ Cy3; D: scatter diagram showing high reproducibility between the replicate experiments of sample $M$. The four subarrays of each microarray were marked, as well as those of the 16 controls. Four differences between $I$ and $M$ samples are marked with arrows. 
Table 1 miRNAs microarray SAM results.

\begin{tabular}{lcccl}
\hline $\begin{array}{l}\text { miRNA } \\
\text { name }\end{array}$ & $\begin{array}{c}\text { Numerator } \\
(r)\end{array}$ & $\begin{array}{c}\text { Denominator } \\
(s+s 0)\end{array}$ & $\begin{array}{c}\text { Fold } \\
\text { change }\end{array}$ & $\begin{array}{c}\text { Localfdr } \\
(\%)\end{array}$ \\
\hline mmu-miR-411 & 6.2812 & 0.1250 & 77.3211 & 0 \\
hsa-miR-495 & 6.1480 & 0.1240 & 70.4865 & 0 \\
mmu-miR-335 & 3.5722 & 0.1374 & 11.7969 & 0 \\
mmu-miR-434-5p & 3.4933 & 0.1941 & 10.9570 & 0 \\
mmu-miR-337 & 3.1466 & 0.2621 & 8.4267 & 2.1369 \\
mmu-miR-379 & 3.0642 & 0.1572 & 8.2660 & 0 \\
mmu-miR-127 & 2.8695 & 0.1050 & 7.2991 & 0 \\
mmu-miR-376a & 2.7728 & 0.0930 & 6.8128 & 0 \\
hsa-miR-181d & 2.5835 & 0.1337 & 5.9634 & 0 \\
mmu-miR-214 & 2.3819 & 0.0883 & 5.2029 & 0 \\
mmu-miR-181c & 2.3210 & 0.1643 & 4.9265 & 0.4391 \\
mmu-miR-361 & 2.0925 & 0.1757 & 4.1878 & 2.2363 \\
mmu-let-7e & 2.0585 & 0.1220 & 4.1362 & 0 \\
mmu-miR-181b & 1.8741 & 0.1129 & 3.6619 & 0 \\
mmu-miR-34a & -1.1681 & 0.0689 & 0.4448 & 3.2707 \\
mmu-miR-29b & -2.4150 & 0.0992 & 0.1871 & 0 \\
mmu-miR-449 & -3.8999 & 0.0625 & 0.0670 & 0.0305 \\
mo-miR-34b & -5.8977 & 0.1917 & 0.0171 & 0 \\
mmu-miR-34c & -6.3315 & 0.1897 & 0.0126 & 0 \\
\hline A & & & & \\
\hline
\end{tabular}

A summary of SAM analysis of $I$ and $M$; fold changes, value of $I / M$; localfdr, local false discovery rates; $q$-value (\%) of all miRNAs was 0.

We compared the expression levels of the $I$ and $M$ samples and ran the PCR products on a 3\% agarose gel. The concordance between relative expression levels and agarose gel analysis was identical on the whole (Fig. 2). To compare the accuracy of efficiency-corrected relative quantification, amplification efficiency was derived from cDNA standard curves. Using the equation mentioned earlier, we calculated the miRNA relative expression levels normalized by U6. The result is shown in Table 2 . Out of 19, 14 miRNAs were detected upregulated and three miRNAs increased slightly in immature mice. Three miRNAs were greatly upregulated, namely mmumiR-411, mmu-miR-495, and mmu-miR-434-5p. The qPCR confirmed the downregulated expression of the five miRNAs identified by microarray in immature mice.

\section{Putative miRNA target gene prediction}

To identify the miRNA-targeting genes, we used the TargetScan program to predict differentially expressed miRNAs corresponding to putative genes (Lewis et al. 2003, Jones-Rhoades \& Bartel 2004, Rajewsky \& Socci
2004). We used PicTar, a computational method for identifying common targets of miRNA (Krek et al. 2005). As a statistical test using genome-wide alignments of vertebrate genomes, PicTar's ability to specifically recover published miRNA targets, and experimental validation of seven predicted targets suggested that PicTar has an excellent success rate in predicting targets for single miRNA and for combinations of miRNA (http://pictar.bio.nyu.edu/).

Transcripts that are directly regulated by miRNA should contain miRNA-binding sites in their 3'-UTRs (Bartel 2004). Binding sites for multiple miRNAs can be found within a UTR, and are frequently repeated within it. Furthermore, conserved binding sites can be found in the 3'-UTRs of homologous genes of related species. We used PicTar to identify common targets of all miRNAs; the result of the analysis is shown in Table 3.

\section{Discussion}

Spermatogenesis is a complex process of germ cell development whereby diploid germ cells proliferate and differentiate into haploid spermatozoa (Cooke \& Saunders 2002). Spermatogenesis occurs in a cyclic manner known as the spermatogenic cycle, which can be divided into 14 stages (I-XIV) on the basis of distinct associations of germ cells (Leblond \& Clermont 1952, de Kretser et al. 1998). There are a few reports on global gene expression in the testis during mouse spermatogenesis. Yu et al. (2003) reported a gene expression study using purified germ cells on a membrane containing 1176 genes, of which only $\sim 200$ were detectable in germ cells.

miRNA regulates gene expression by binding and modulating the translation of specific mRNAs. Several hundred miRNAs have been verified currently, however, thousands of miRNA genes in various genomes still need to be identified (Ruvkun et al. 2004). Even in mammalian, there are still many miRNAs waiting to be detected and only little is known about miRNA expression levels or patterns in spermatogenesis as well. Therefore, it is necessary to study miRNA relating to spermatogenesis using microarray and qPCR technologies.

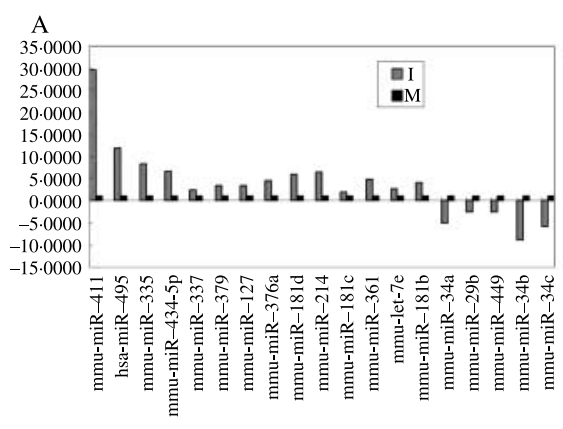

B

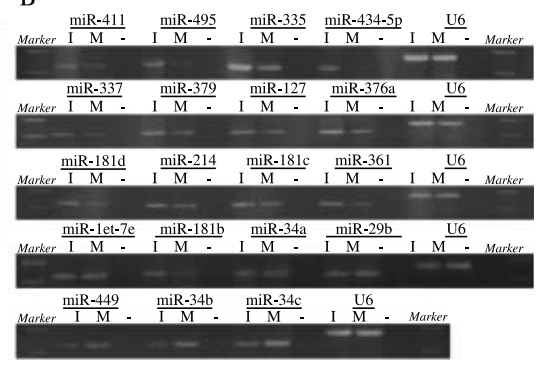

Figure 2 miRNA expression in mouse testis was identified by qPCR. A: The histogram of the relative expression levels between $I$ and $M$ samples, 19 miRNAs are listed on the $x$-axis, the $y$-axis refers to the relative expression levels between $I$ and $M$ samples, and the $M$ sample expression is 1 . B: The electrophoresis result of $\mathrm{PCR}$ products, $\mathrm{U} 6$ is used as internal control, marker, 50 bp DNA ladder; (-), negative control; $I$, immature testes tissue; and $M$, mature testes tissue. 
Table 2 Results carried out by qRT-PCR.

\begin{tabular}{lcl}
\hline miRNA name & Efficiency & Fold change $^{\text {a }}$ \\
\hline U6 & 1.792 & 1 \\
mmu-miR-411 & 1.819 & 29.4872 \\
hsa-miR-495 & 1.804 & 11.9202 \\
mmu-miR-335 & 1.802 & 8.282 \\
mmu-miR-434-5p & 1.792 & 6.6704 \\
mmu-miR-337 & 1.85 & 2.3589 \\
mmu-miR-379 & 1.737 & 3.3764 \\
mmu-miR-127 & 1.827 & 3.2921 \\
mmu-miR-376a & 1.816 & 4.5472 \\
hsa-miR-181d & 1.855 & 6.0445 \\
mmu-miR-214 & 1.849 & 6.5125 \\
mmu-miR-181c & 1.76 & 1.9309 \\
mmu-miR-361 & 1.747 & 4.7487 \\
mmu-let-7e & 1.77 & 2.6322 \\
mmu-miR-181b & 1.776 & 4.1412 \\
mmu-miR-34a & 1.76 & 0.1975 \\
mmu-miR-29b & 1.749 & 0.4035 \\
mmu-miR-449 & 1.745 & 0.3859 \\
rno-miR-34b & 1.807 & 0.1121 \\
Mmu-miR-34c & 1.817 & 0.1745 \\
\hline All Civalues & & \\
\hline
\end{tabular}

All $C_{\mathrm{t}}$ values have been averaged from three repeated experiments. Amplification efficiency was derived from cDNA standard curves.

${ }^{a}$ Fold changes $(I / M)$ displayed above had been all normalized by $U 6$ as reference gene.

miRNA microarray provides a parallel and highthroughput method of detecting thousands of miRNAs simultaneously. Quantitative real-time PCR is the golden standard for gene expression quantification (Livak \& Schmittgen 2001, Peirson et al. 2003). Now, stem-loop RT primers have better specificity and sensitivity than conventional linear ones likely due to the base stacking and spatial constraint of the stem-loop structure (Chen et al. 2005). The qRT-PCR data correlated well with microarray analysis and demonstrated the reliability of SAM assay.

The first miRNA, lin-4, was identified in 1993. Till October 2006, Sanger miRNAs Release 9.0 database contains 4361 entries representing hairpin precursor miRNAs, expressing 4167 mature miRNA products. But, there is a large pool of miRNA sequences yet to be discovered. In this study, three miRNAs were found which had no corresponding mouse miRNAs but were expressed differently between $I$ and $M$ samples. These miRNAs were further confirmed by qPCR experimentally, which indeed expressed differently between immature and mature mouse testes and its target genes could play a role in spermatogenesis. Mouse corresponding miRNA cloning based on human or rat miRNA sequences and its biological function need further studies.

The miRNA microarray results show that sexually immature mouse testis contained a range of miRNAs at higher expression levels than mature tissue. Computational methods were used to predict the target genes of those miRNAs. Genes associated with mammalian development and spermatogenesis identified by prediction and the data are displayed in Table 3. One of these genes is $\mathrm{Brd} 2$, targeted by mmu-miR-127; $\mathrm{Brd} 2$ was observed in a previous study expressed at high levels in diplotene spermatocytes and round spermatids while at low levers in spermatogonia. In situ hybridization and immunostaining on histological sections of mouse testes revealed a strikingly specific and dynamic change of cellular specificity in the germ line during the progression of spermatogenesis. The expressing patterns of mmu-miR-127 performs are correlated to $\mathrm{Brd} 2$ and this strongly suggests a close relationship between the identified miRNA and its target gene.

Among the predicted target genes, Usp42 gene has been reported to express during embryogenesis and spermatogenesis in the mouse (Kim et al. 2006), predictions associate this gene with mmu-miR-411 and mmu-miR-29b. Rsbn1 gene, a novel homeobox-like protein gene is expressed exclusively in round spermatids and plays an important role in transcriptional regulation in haploid germ cells (Takahashi et al. 2004), is predicted by some miRNAs. Edn1 is produced by and biologically active in the testes of several mammals (Maggi et al. 1995). Sox5 and Sox6 genes

Table 3 Target genes for miRNAs.

\begin{tabular}{|c|c|c|c|}
\hline miRNA name & Chromosome location & Fold change & Putative targets \\
\hline mmu-miR-411 & 12 & 77.3211 & Usp42 \\
\hline mmu-miR-335 & 6 & 11.7969 & Ccnt2, CcnD2, Rsbn1 \\
\hline mmu-miR-434-5p & 12 & 10.957 & N/A \\
\hline mmu-miR-337 & 12 & 8.4267 & Ybx1, Ap1g1 Taf5, Taf12, Creb1, Ccnl1 \\
\hline mmu-miR-379 & 12 & 8.266 & Eif4g2, Edn1, Rnf6 \\
\hline mmu-miR-127 & 12 & 7.2991 & $\mathrm{Brd} 2$ \\
\hline mmu-miR-376a & 12 & 6.8128 & N/A \\
\hline mmu-miR-214 & 1 & 5.2029 & Hspd1, Tex27, Adcyap1r1, Hbp1, Ap1g1, Ssr1 \\
\hline mmu-miR-181c & 8 & 4.9265 & Sox6, Sox5, Rsbn1, Adm, Tnpo1, Dazap2, Kpnb1 \\
\hline mmu-miR-361 & $x$ & 4.1878 & Kpnb1, Zfp148, Bmpr2, Calm2 \\
\hline mmu-let-7e & 17 & 4.1362 & Nr6a1, Taf5, Fasl, Eif4g2, Suv39h2, Dzip1, Ddx19b \\
\hline mmu-miR-181b & 1 & 3.6619 & Tbp11, Rsbn1, Adm, Dazap2, Pik3r3, Rnf6 \\
\hline mmu-miR-34a & 4 & 0.4448 & $C c n D 2, B c / 2, G m f b$ \\
\hline mmu-miR-29b & 6 & 0.1871 & Creb5, Bak1, Usp42, Mlf1, Hbp1, Snx24 \\
\hline mmu-miR-449 & 13 & 0.067 & Sox 11, CcnE2, Bcl2, Gmfb \\
\hline mmu-miR-34c & 11 & 0.0126 & SPAG4, Ccnl1, Zfp148, Gmfb \\
\hline
\end{tabular}


belong to the Sox family that are highly expressed in adult mouse testes, the HMG (high mobility group) domains of both proteins bind to the sequence $5^{\prime}$-AACAAT-3', suggesting that these two genes may have overlapping functions in the regulation of gene expression during spermatogenesis in adult mice (Denny et al. 1992, Connor et al. 1995). NR6A1 (also called germ cell nuclear factor (GCNF)), an orphan member of the nuclear receptor gene superfamily, expresses predominantly in developing germ cells of the adult mouse (Chen et al. 1994, Hirose et al. 1995, Katz et al. 1997). NR6A1 played a role in transcriptional regulation during meiosis and the early haploid phase of spermatogenesis (Yang et al. 2003).

In conclusion, we conduct microarray assay and quantitative real-time PCR to examine miRNA expression in mouse testes between sexually immature and mature individuals, and obtain 19 miRNAs significant expressing differently in immature and mature tissues. Next step we will continue to study these miRNAs function.

\section{Acknowledgements}

We thank Dr Xianming Mo (Lab of Hematology, West China Hospital) for many helpful discussions during this work and critical reading of the manuscript. We further thank Prof. Xuyang Liu (Laboratory of Ophthalmology, West China Hospital) and Stethun Altwood (Center for Biotherapy of Cancer and Cancer Research Center, West China Hospital) for critical review of the manuscript. This work was supported by Special Funds for Major State Basic Research Project of China (grant no. 90408025, 30200153, 30500186). The authors declare that there is no conflict of interest that would prejudice the impartiality of this scientific work.

\section{References}

Bartel DP 2004 MicroRNAs: genomics, biogenesis, mechanism, and function. Cell 116 281-297.

Braun RE 1998 Post-transcriptional control of gene expression during spermatogenesis. Seminars in Cell and Developmental Biology 9 483-489.

Calin GA, Liu CG, Sevignani C, Ferracin M, Felli N, Dumitru CD, Shimizu M, Cimmino A, Zupo S, Dono M, Marie L, Aquila D, Alder H, Rassenti L, Kipps TJ, Bullrich F, Negrini M \& Croce CM 2004 MicroRNA profiling reveals distinct signatures in B cell chronic lymphocytic leukemias. PNAS 101 11755-11760.

Chen F, Cooney AJ, Wang Y, Law SW \& O'Malley BW 1994 Cloning of a novel orphan receptor (GCNF) expressed during germ cell development. Molecular Endocrinology 8 1434-1444.

Chen C, Ridzon DA, Broomer AJ, Zhou Z, Lee DH, Nguyen JT, Barbisin M, Xu NL, Mahuvakar VR, Andersen MR, Lao KQ, Livak KJ \& Guegler KJ 2005 Real-time quantification of microRNAs stemloop RT-PCR. Nucleic Acids Research 33 e179.

Connor F, Wright E, Denny P, Koopman P \& Ashworth A 1995 The Sryrelated HMG box-containing gene Sox6 is expressed in the adult testis and developing nervous system of the mouse. Nucleic Acids Research 23 3365-3372.

Cooke HJ \& Saunders PT 2002 Mouse models of male infertility. Nature Reviews. Genetics 3 790-801.
Denny P, Swift S, Connor F \& Ashworth A 1992 An SRY-related gene expressed during spermatogenesis in the mouse encodes a sequence-specific DNA binding protein. EMBO Journal 11 3705-3712.

He H, Jazdzewski K, Li W, Liyanarachchi S, Nagy R, Volinia S, Calin GA, Liu CG, Franssila K, Suster S, Kloos RT, Croce CM \& de la Chapelle A 2005 The role of microRNA genes in papillary thyroid carcinoma. PNAS 102 19075-19080.

Hirose T, O'Brien DA \& Jetten AM 1995 RTR: a new member of the nuclear receptor superfamily that is highly expressed in murine testis. Gene 152 247-251.

Jones-Rhoades MW \& Bartel DP 2004 Computational identification of plant microRNAs and their targets, including a stress-induced miRNA. Molecular Cell 14 787-799.

Katz D, Niederberger C, Slaughter GR \& Cooney AJ 1997 Characterization of germ cell-specific expression of the orphan nuclear receptor, germ cell nuclear factor. Endocrinology 138 4364-4372.

Kim YK, Kim YS, Yoo KJ, Lee HJ, Lee DR, Yeo CY \& Baek KH 2006 The expression of Usp42 during embryogenesis and spermatogenesis in mouse. Gene Expression Patterns 7 143-148.

Krek A, Grun D, Poy MN, Wolf R, Rosenberg L, Epstein EJ, MacMenamin P, da Piedade I, Gunsalus KC, Stoffel M \& Rajewsky N 2005 Combinatorial microRNA target predictions. Nature Genetics 37 495-500.

de Kretser DM, Loveland KL, Meinhardt A, Simorangkir D \& Wreford N 1998 Spermatogenesis. Human Reproduction 13 1-8.

Kuramochi-Miyagawa S, Kimura T, Ijiri TW, Isobe T, Asada N, Fujita $Y$, Ikawa M, Iwai N, Okabe M, Deng W, Lin H, Matsuda Y \& Nakano T 2004 Mili, a mammalian member of piwi family gene, is essential for spermatogenesis. Development 131 839-849.

Leblond CP \& Clermont Y 1952 Definition of the stages of the cycle of the seminiferous epithelium in the rat. Annals of the New York Academy of Sciences $\mathbf{5 5}$ 548-573.

Lee RC, Feinbaum RL \& Ambros V 1993 The $C$. elegans heterochronic gene lin-4 encodes small RNAs with antisense complementarity to lin-14. Cell 75 843-854.

Lewis BP, Shih IH, Jones-Rhoades MW, Bartel DP \& Burge CB 2003 Prediction of mammalian microRNA targets. Cell 115 787-798.

Livak KJ \& Schmittgen TD 2001 Analysis of relative gene expression data using real-time quantitative PCR and the $2(-\Delta \Delta \mathrm{C}(\mathrm{T}))$ method. Methods 25 402-408.

Maggi M, Barni T, Orlando C, Fantoni G, Finetti G, Vannelli GB, Mancina R, Gloria L, Bonaccorsi L, Yanagisawa M \& Forti G 1995 Endothelin-1 and its receptors in human testis. Journal of Andrology 16 213-224.

Peirson SN, Butler JN \& Foster RG 2003 Experimental validation of novel and conventional approaches to quantitative real-time PCR data analysis. Nucleic Acids Research 31 e73.

Rajewsky N \& Socci ND 2004 Computational identification of microRNA targets. Developmental Biology 267 529-535.

Ruvkun G, Wightman B \& Ha I 2004 The 20 years it took to recognize the importance of tiny RNAs. Cell 116 93-96.

Schmittgen TD, Jiang J, Liu Q \& Yang L 2004 A high-throughput method to monitor the expression of microRNA precursors. Nucleic Acids Research 32 e43.

Takahashi T, Tanaka H, Iguchi N, Kitamura K, Chen Y, Maekawa M, Nishimura $H$, Ohta $H$, Miyagawa $Y$, Matsumiya $K$, Okuyama A \& Nishimune Y 2004 Rosbin: a novel homeobox-like protein gene expressed exclusively in round spermatids. Biology of Reproduction 70 1485-1492.

Thomson JM, Parker J, Perou CM \& Hammond SM 2004 A custom microarray platform for analysis of microRNA gene expression. Nature Methods 1 47-53.

Tusher VG, Tibshirani R \& Chu G 2001 Significance analysis of microarrays applied to the ionizing radiation response. PNAS $\mathbf{9 8}$ 5116-5121. 
Wienholds E, Kloosterman WP, Miska E, Alvarez-Saavedra E, Berezikov E, de Bruijn E, Horvitz RH, Kauppinen S \& Plasterk RH 2005 MicroRNA expression in zebrafish embryonic. Developmental Science 309 310-311.

Xie X, Lu J, Kulbokas EJ, Golub TR, Mootha V, Lindblad-Toh K, Lander ES \& Kellis M 2005 Systematic discovery of regulatory motifs in human promoters and $3^{\prime}$ UTRs by comparison of several mammals. Nature 434 338-345.

Yang G, Zhang YL, Buchold GM, Jetten AM \& O'Brien DA 2003 Analysis of germ cell nuclear factor transcripts and protein expression during spermatogenesis. Biology of Reproduction 68 1620-1630.

Yu Z, Guo R, Ge Y, Ma J, Guan J, Li S, Sun X, Xue S \& Han D 2003 Gene expression profiles in different stages of mouse spermatogenic cells during spermatogenesis. Biology of Reproduction 69 37-47.
Yu Z, Raabe T \& Hecht NB 2005 MicroRNA Mirn122a reduces expression of the posttranscriptionally regulated germ cell transition protein 2 (Tnp2) messenger RNA (mRNA) by mRNA cleavage. Biology of Reproduction 73 427-433.

Zhao Y, Samal E \& Srivastava D 2005 Serum response factor regulates a muscle-specific microRNA that targets Hand2 during cardiogenesis. Nature 436 214-220.

Received 6 December 2006

First decision 4 January 2007

Revised manuscript received 6 March 2007

Accepted 29 March 2007 